\title{
'Verstehen' heißt nicht 'mögen'
}

Theo Harden (Dublin)

\begin{abstract}
:
'Verstehen' heißt nicht 'mögen'

The discussion in the entire area of "intercultural communication" revolves around the issue of facilitating mutual understanding between members of ethnic and cultural communities. The basic assumption is that once this has been achieved, the fundamental problems have been addressed, thereby removing the obstacles for sympathetic interaction. The conclusion that understanding is a necessary condition for liking, is, however, faulty. Empathy and sympathy are - even though related - not identical. In this contribution I will argue that getting to know the "other" better will not automatically result in liking him/her more. The history of exotism from Marco Polo via Columbus to even such sober characters as James Cooke shows that phenomena which are not understood may create very strong positive feelings whereas in an intracultural setting which is certainly not ridden by difficulties encountered in intercultural exchange, we do find clearly marked antipathies. A further topic to be addressed is the issue of whether the matters in question are intrinsically accessible and capable of being mediated in formal instruction.
\end{abstract}

\section{$1 \quad$ Fremdheit}

Irgendwo fremd zu sein ist ein Zustand, der bis ins 20. Jahrhundert als wenig erstrebenswert galt (vgl. Enzensberger 1968: 149 ff). Fremde waren den Einheimischen suspekt und dementsprechend verhielten diese sich auch. Zwar existierten durchaus bestimmte, das Gastrecht betreffende Regeln, aber für deren Einhaltung gab es nicht unbedingt eine Gewähr. Der Konflikt zwischen eher naturwüchsiger Ablehnung (die bis zu Raub, Mord und Vergewaltigung gehen konnte) einerseits und eher konventionell geregelter freundlicher Aufnahme des Fremden (zumindest für einen bestimmten Zeitraum), wird prägnant in Genesis 19,1ff beschrieben. Der Ort: Sodom. Zwei Männer kommen dort spät abends an und werden von Lot in sein Haus gebeten. Nachdem ihre Ankunft sich herumgesprochen hat, versammeln sich Sodoms Bürger vor Lots Haus und verlangen von ihm, daß er seine Gäste herausgibt: Sie riefen nach Lot und fragten ihn:

"Wo sind die Männer, die heute Abend zu dir gekommen sind? Heraus mit ihnen, wir wollen mit ihnen verkehren" (Genesis 19,5). Verkehren ist hier im Sinne von 'Geschlechtsverkehr haben' zu verstehen.

Lot versucht den Konflikt zwischen dem Bruch des Gastrechts und dem ihm offensichtlich nicht völlig unverständlichen Wunsch seiner Mitbürger, die Fremden zu mißhandeln, dadurch zu lösen, daß er als Kompromiß seine jungfräulichen Töchter zur Vergewaltigung anbietet. 
Das wohlverdiente Ende der Stadt und ihrer Bürger dürfte bekannt sein: bei den Fremden handelte es sich um Engel, die, erzürnt über das, was ihnen widerfahren ist, Gott veranlaßten, Feuer und Schwefel regnen zu lassen.

In diesen wenigen Zeilen aus Genesis kann man - mit etwas Kreativität - bereits die beiden Extrempositionen von Fremdwahrnehmung finden: Ablehnung die in letzter Konsequenz in Vernichtung mündet und Akzeptanz bis zur Selbstaufgabe. In abgemilderter Form sind sie als Xenophobie und Exotismus bekannt. Ein Merkmal, das beide Positionen miteinander gemein haben, ist die inadequate Wahrnehmung des Fremden. Dämonisierung hier, Idolisierung dort. Wie eingangs angedeutet wurde, hat sich die Einstellung zum Fremdsein im Verlaufe des 20. Jahrhunderts deutlich geändert. Einerseits durch eine immer rascher voranschreitende Internationalisierung, die unter anderem auch an die sprunghaften Fortschritte in der Nachrichtenübermittlungstechnik gekoppelt war, andereseits durch den Tourismus. Der erste Faktor förderte in immer stärkerem Maße die Migrationsbewegungen von Arbeitskräften, die dann, dort wo sie ankamen eben Fremde waren, der zweite bot immer breiteren Schichten die Möglichkeit, sich ungefährdet für einen begrenzten Zeitraum dem wohligen Schauer des Fremdseins zu überlassen. ${ }^{1}$ Beide führten aber - sowohl in der gewohnten Umwelt als auch in der bewußt gesuchten Fremde - für immer weitere Kreise zu einer Erfahrung, die bis dahin auf wenige spezialisierte Gruppen beschränkt war: Fremderfahrung. Kommunikation zwischen Angehörigen verschiedener Kulturen fand zwangsläufig statt, häufig war sie aber wenig erfolgreich, bzw. nicht so erfolgreich, wie sie hätte sein können.

Es ist daher nur konsequent, wenn im Rahmen dieser Entwicklung die interkulturelle Kommunikation im Sinne eines gelungenen Austausches mit dem Anderen, als Problem identifiziert wurde.

Das sich formierende global village erfordert, daß die Störanfälligkeit dieses Typs Kommunikation wenn schon nicht behoben, so doch deutlich reduziert wird. Mißkommunikation, das machen Autoren wie Chen/Starosta (1997: 4ff) mit erfrischender Deutlichkeit klar, können wir uns nicht leisten. Vorfälle wie jener in Sodom sollen unter allen Umständen vermieden werden, denn sie behindern nicht nur das friedliche Zusammenleben, sie verhindern - was vermutlich schwerer wiegt - den reibungslosen globalen Austausch von Waren und Dienstleistungen.

Trotz der sowohl aus moralisch-ethischer wie aus ökonomischer Perspektive löblichen Zielsetzung ist das Konzept der interkulturellen Kommunikation von den verschiedensten Positionen aus immer wieder heftig angegriffen worden (vgl. Hu 2000: 75ff), wobei Volker Hinnenkamps Beitrag Von den Schwierigkeiten (mit) der Interkulturellen Kommunikation (Hinnenkamp 1994) wegen seiner Ausgewogenheit und grundsätzlich konstruktiven Tendenz als beispielhaft für die Diskussion innerhalb dieses Forschungsfeldes angesehen werden kann. Hinnenkamp vertritt die Auffassung, daß ungeachtet aller Übertreibungen, Verzerrungen und völlig unangemessener Ansprüche, mit denen das Konzept der interkulturellen Kommunikation belastet ist, ein Verstehen des Anderen und ein Akzeptieren seines Andersseins grundsätzlich die Möglichkeiten für einen Interessenausgleich schaffen, daß es also durchaus lohnt, in dieser Richtung weiter zu arbeiten und zu forschen.

\footnotetext{
${ }^{1}$ Völkerwanderungen sind kein Phänomen des 20. Jahrhunderts. Im Unterschied zu früheren Zeiten sind die modernen Bewegungen jedoch in den seltensten Fällen mit Okkupation oder Landnahme und den daraus resultierenden gewaltsamen Auseinandersetzungen verbunden. Eine soziale Stratifikation findet dementsprechend auch nicht in der Form Sieger - Besiegte statt, sondern ist vielmehr sehr stark an den wirtschaftlichen Erfolg der Individuen gekoppelt.
} 
Seine Einschätzung, die weitestgehend von der sich mit diesem Thema befassenden scientific community geteilt wird, basiert auf der Annahme, daß durch das Verstehen und die sich damit erweiternden Möglichkeiten, sich in den Anderen hineinzuversetzen, dessen Rolle sympathetisch interpretiert wird. Wenn ich den Anderen verstehe, dann kann ich kaum umhin, ihn auch zu mögen, dies scheint die Maxime zu sein, die - ungeprüft und stillschweigend weitgehend akzeptiert wird. Die folgenden Überlegungen beschäftigen sich mit dieser Annahme, also mit der tendenziellen Gleichsetzung von 'Empathie' und 'Sympathie' und ihrem Einfluß auf die einschlägige Forschung.

\section{$2 \quad$ Empathie}

Empathie, landläufig als Einfühlungsvermögen definiert, trägt in aller Regel die Konnoation 'positives Einfühlungsvermögen'. Krappmann (1978: 142) unterstreicht zwar, daß es sich bei Empathie in der Mead'schen Definition um eine grundsätzlich kognitive Fähigkeit handele, die kein Wohlwollen gegenüber dem Anderen voraussetzt; aber bereits auf der nächsten Seite wird das Konzept modifiziert und die Wichtigkeit der positiven emotionalen Komponente herausgestellt. Empathiefähigkeit in diesem postitiven Sinne ist daher auch eines der Hauptanliegen interkulturellen Kommunikationstrainings. Man betrachte dazu nur die von Harris/Moran (1989) formulierten "five attitudinal imperatives" oder die von Kale (1994: 438f) in einem Beitrag mit dem programmatischen Titel Peace as an Ethic for Intercultural Communication postulierten - ethischen - Prinzipien. Der sich aufdrängende Eindruck ist, daß immer, wenn von Empathie die Rede ist, eigentlich Sympathie gemeint wird. Daß es sich aber hierbei nicht um deckungsgleiche Konzepte handelt, ist bereits von Heider (1958: 277ff) herausgearbeitet worden. Zwar, so Heider, handelt es sich bei Empathie und Sympathie durchaus um universale menschliche Fähigkeiten, sie müssen jedoch hinsichtlich ihrer Konsequenzen für das jeweilige Individuum und den/die in Rede stehenden Anderen differenziert werden. Aufbauend auf dieser Argumentation analysiert Benson (2001: 149ff) interpersonales Verhalten sowohl von Mitgliedern einer bestimmten Gruppe untereinander als auch von Angehörigen verschiedener Gruppen und kommt dabei zu dem Schluß:

The capacity for sympathy is universal, as is that for empathy, but the objects of sympathy are local in the
same way that the capacity for fear or disgust is universal but their objects are local and variable. Worms
may disgust us as a culinary delicacy but may delight others. Therefore, on this hypothesis, it is not that
the natural capacity for sympathy is inhibited, but rather that particular unsympathised objects have not
been included in the category of the sympathetic when those categories were being constructed and filled.
The connection between empathy and sympathy must be actively constructed, rather than being naturally
there already... . In extreme circumstances empathy may be fully in play in that an aggressor or torturer
knows full well what it is like to be the victim and understands with Sadean precision how the world
looks to the victim, but that sympathy is simply not in play at the time. (Benson 2001: 152) Angewandt auf interkulturelle Kommunikation hieße dies nun, daß - entgegen der Annahme ihrer Apologeten - möglicherweise gar nicht im Bereich der Empathie nach den Ursachen möglicher Mißkommunikation gesucht werden muß, sondern vielmehr in der bewußten und gewollten Abgrenzung von Gruppen untereinander. Dieser Prozeß ist durchaus auch innerhalb intrakultureller Kommunikation anzutreffen, denn, so Schlesinger (1987: 335): 
...identity is as much about exclusion as it is about inclusion, and the critical factorfor defining the ethnic group therefore becomes the social boundary which defines the group with respect to other groups ...not the cultural reality within its borders. (Hervorhebung im Original, T.H.)

Die dergestalt als notwendig empfundene Grenzziehung nimmt bisweilen - und dies scheint ein weltweit verbreitetes, nicht auf Europa beschränktes Phänomen zu sein - drastische Formen an, wie Todorov (1984: 76) pointiert bemerkt:

It is in this fashion that European Slavs call their German neighbours nemec, 'mutes', the Mayas ... call the Toltec nunob, 'mutes' ... and the Aztecs ... call those who do not speak Nahuatl tenime, 'barbarians' or popoloca, 'savages', because they share the scorn of all peoples for their neighbours.

Die Tatsache, daß es sich immer um Nachbarvölker handelt, stimmt bedenklich. Unüberwindliche natürliche Hindernisse können kaum für diese problematische Fremdwahrnehmung verantwortlich gemacht werden. Der Kontakt war ja durchaus vorhanden, er wurde aber offensichtlich nicht als Bereicherung empfunden. Interkulturelle Kommunikation fand nicht statt, wobei kaum anzunehmen ist, daß ausschießlich die unterschiedlichen sprachlichen Zeichen der Grund dafür sind. Eher leuchtet da Donalds Erklärung ein:

Manifest racism, its violent misognyny, and its phobias about alien cultures, alien ideologies and 'enemies within', is the terror that without the known boundaries, everything will collapse in undifferentiated, miasmic chaos, that identity will disintegrate and the 'I' will be suffocated or swamped. ${ }^{2}$ (Donald 1988:44)

Diese Befürchtungen, so verständnisvoll sie auch zur Kenntnis genommen werden, sind genau das, was vermittels interkutureller Kommunikation aus der Welt geschafft werden soll. Es lohnt daher, an dieser Stelle etwas genauer zu beleuchten, wie dieser Prozeß gesehen wird, denn er soll ja therapeutisch vorwiegend gegen ethnische Grenzziehungen, bzw. die daraus resultierenden - weitgehend als irrational und überflüssig empfundenen - Konflikte wirken. ${ }^{3}$ Aussagen wie: "Living is largely a matter of communication" (Smith 1966: 1), "Everything human beings do may, one way or another, be subsumed under the general heading of 'communication'" (Gordon 1969: IX) oder auch das immer noch populäre, von Watzlakwick postulierte dictum, daß man nicht 'nicht kommunizieren' kann, zeigen, daß das Konzept 'Kommunikation' recht umfassend interpretiert werden kann, allerdings in seiner gesamten Vielschichtigkeit auch recht vage und flexibel bleibt.

Kommunikation also als ultima ratio, wobei vor allem die verbale Interaktion im Vordergrund steht, unter anderem wohl auch, weil sich dieses Element des komplexen Gesamtbereichs 'Kommunikation' einem analytischen Zugriff am wenigsten verschließt. Zu der Annahme, daß Kommunikation in all ihren Ausprägungen und Ebenen letztendlich das Fundament jeder Sozialstruktur bildet gibt es kaum eine sinnvolle Alternative. Die Frage, ob daraus abgeleitet werden kann, daß durch kommunikative Kompetenz auch 'irrationale'

\footnotetext{
${ }^{2}$ Vgl. dazu auch Ricoeur (1965:278): “...when we discover that there are several cultures, instead of just one, and consequently, at the time we acknowledge the end of a sort of cultural monoply, be it illusory or real, we are theatened ... [by] our own discorvery. Suddenly, it becomes possible that there are just Others, that we ourselves are an 'other' among Others." (zit. nach Morley/Robinson (1995: 25).

${ }^{3}$ Gegen welche fundamentalen Befindlichkeiten und strukturellen Probleme interkulturelle Kommunikation zu arbeiten hat, wird in dem ausgezeichneten Beitrag von von Hartmut Esser (1996) in bemkerkenswerter Klarheit herausgearbeitet. Der ,gnadenlose Wettlauf“ um „Ressourcen mit Positionsguteigenschaften ... ist durch einfache Absprachen nicht zu bändigen. Die Versuchung, die Absprachen nicht einzuhalten, sind viel zu groß, als daß man sich auf eine bloße ... Verständigung verlassen könnte.“ (Esser 1996: 79f)
} 
Konflikte vermieden bzw. entschärft werden können ist jedoch $\mathrm{m}$. E. bisher nicht überzeugend beantwortet worden. Die sogenannte 'kommunikative Wende' im Fremdsprachenunterricht, auf deren historische Entwicklung und Einordnung hier verzichtet werden kann, hatte jedoch ungeachtet dessen nicht 'nur' das Erreichen sprachlicher, sondern kommunikativer Kompetenz zum Ziel, was vor allem im Rahmen institutionellen Unterrichts ganz besonders interessante Folgeerscheinungen gezeitigt hat. Vom Gegenstand her waren die Entwicklungen, die durch diese Wende eingeleitet wurden, logischerweise in Richtung 'interkultureller Kommunikation' und 'interkultureller Kompetenz' angelegt. Das Verstehen des 'Anderen' und das Verstandenwerden durch den 'Anderen' wurden als die zu erreichenden Zielgrößen identifiziert, wobei das jeweilige 'Anderssein' vorzugsweise kulturell, sprachlich oder ethnisch gefaßt wurde, was natürlich ein Verstehen und Verstandenwerden objektiv zumindest im eher technischen Bereich der schieren Informationsübermittlung - erschwert.

Betrachtet man Kommunikation allgemein und verbale Interaktion speziell in Anlehnung an Leech (1983: 36ff) als eine geordnete Menge von Problemlösungsstrategien, dann liegt die Aufgabe der an der Kommunikation Beteiligten darin, eine means-end Analyse vorzunehmen. ${ }^{4}$ D. h. sie müssen sich fragen, welche kommunikativen Akte das Erreichen eines bestimmten Zieles am wahrscheinlichsten erscheinen lassen. In aller Regel geht nun die Forschung davon aus, daß das Ziel eines kommunikativen Prozesses von allen Beteiligten kooperativ angestrebt wird. Wo dies nicht geschieht, treten Probleme auf, es müssen Reparaturmaßnahmen eingeleitet werden, und möglicherweise bricht die Kommunikation völlig zusammen. Ein deartiger Kommunikations-GAU kann jedoch, so die Annahme, nicht im Interesse der Beteiligten sein, folglich werden sie alles daran setzen, ihn zu vermeiden. Vor diesem Hintergrund ist der größte Teil der kommunikationsanalytischen Forschung zu sehen. Mißkommunikation wird als Störung betrachtet, deren Auftreten man tunlichst vermeidetet (vgl. auch Coupland/Giles/Wiemann 1991). Daß das Mißlingen von Kommunikation nicht notwendigerweise auf der kommunikativen Inkompetenz eines oder mehrerer Akteure beruhen muß, sondern ganz bewußt herbeigeführt werden kann, und daß die eingesetzten Mittel damit unter die oben angeführten Problemlösungsstrategien fallen, wird weitestgehend außer Betracht gelassen. Ein Verhalten, durch das die Kommunikation erschwert oder völlig unmöglich gemacht wird, gilt als eher pathologisch und dementsprechend therapiebedürftig. ${ }^{5}$ Mit anderen Worten: es wird allgemein davon ausgegangen, daß Kommunikation im Sinne einer positiv angewandten Strategie zur uncertainty reduction funktioniert, wobei die Reduktion der Ungewissheit als Wissenserweiterung hinsichtlich der uns umgebenden Anderen und unserer selbst verstanden werden muß (vgl Berger/Bradac 1982: 5ff). Was unbeachtet bleibt, ist, daß für derart positive Szenarien eine Intention der Beteiligten vorausgesetzt werden muß, deren Ziel in eben jener 'uncertainty reduction' besteht. Aber ist diese Annahme eigentlich gerechtfertigt? Oder, mit Blick auf die 'interkulturelle Kompetenz' gefragt: führt diese, selbst wenn sie erreicht wird

\footnotetext{
${ }^{4}$ Vgl. dazu die ausgesprochen anregenden Arbeiten von Winston (1977) und Parisi/Castelfranchi (1981)

${ }^{5}$ Wie hoch der Stellenwert von Kommunikation häufig eingeschätzt und wohl auch überschätzt wird, kann exemplarisch durch das folgende Zitat illustriert werden: "The ability, through increased awareness and understanding, to coexist peacefully with people who do not necessarily share our backgrounds, beliefs, values, of life styles can not only benefit us in our own neighborhoods but can alos be a decisive factor in forestalling nuclear annihilation." (Samovar/Porter 1988: 1f)
} 
(was ja letztlich bedeutet, dass man in einem interkulturellen Kontext kommunikativ eben so kompetent agiert wie im intrakulturellen), dazu, dass der kulturell 'Andere' dann auch notwendigerweise positiv wahrgenommen wird?

Weiter oben wurde gesagt, daß kommunikatives Handeln als eine geordnete Menge von Problemlösungsstrategien verstanden werden soll. Außerdem soll als Ziel dieses Handelns die Reduktion von Unsicherheit bezüglich des bzw. der jeweils anderen angenommen werden. Des weiteren versichert kommunikatives Handeln einerseits die Beteiligten der Gültigkeit der sozialen Realität und schafft andererseits jeweils neue Realitäten. Ohne ein gewisses Maß an Empathiefähigkeit ist dieser Prozeß nicht denkbar, denn auch das bewußte Herbeiführen eines Scheiterns bedarf ja der antizipierenden Rollenübernahme. Oder anders: die der Kooperation zu Grunde liegenden Regeln müssen an der strategisch entscheidedenden Stelle verletzt werden. Eine Beleidiung verfehlt ja auch ihr Ziel, wenn ihr Objekt sich nicht beleidigt fühlt.

Was ist aber genauer unter dieser 'Empathiefähigkeit' zu verstehen? Folgt man der konstruktivistischen Systemtheorie, dann muß das menschliche Nervensystem als selbstreferentiell und operational geschlossen gesehen werden (vgl. Roth 1987: 235). Daraus folgt, daß sich die kognitiven Bereiche der Individuen nicht überschneiden. Die Interaktion geschlossener Systeme, die ja unter diesen Annahmen eigentlich nicht möglich ist, findet nach Maturana (1982) jedoch dann statt, wenn strukturelle Ähnlichkeiten vorliegen. In diesem Falle lösen diese Systeme wechselseitig selbstrefentielle Prozesse aus. Sie entwickeln sich parallel, ohne daß es dazu eines Informationsaustauschs bedürfte. Der gesamte soziale Bereich kann als fortdauernder Prozeß solcher struktureller Kopplungen gesehen werden (vgl. Hejl 1987), wobei die Parallelisierung dementsprechend dazu führt, daß Ereignisse vergleichbare Interpretationen erfahren. Empathiefähigkeit kann somit gemessen werden am Grad der Parallelisierung, die die Individuen herzustellen in der Lage sind.

Dies muß jedoch differenziert werden, denn einerseits handelt es sich zweifelsohne um ein anthropologische Konstante: Der weiter oben angeführte Folterknecht kann jedes menschliche Wesen erfolgreich foltern, da er sich auf einer grundsätzlichen Ebene immer in sein Opfer hineinzufühlen vermag. Andererseits zeigt jedoch die Tatsache, daß überhaupt kommuniziert wird, daß die Parallelisierung jenseits des Kreatürlichen ein dynamischer Prozeß ist, der nach Schmidt (1992: 305) die engen Beziehungen zwischen Kognition (als Konstruktuion der Realität), Interaktion (als Abgleich der verschiedenen Realitätskonstruktionen) und Institutionen (als Organisationseinheiten der Interaktion) vermittelt.

Die Regeln, nach denen dies geschieht, sind innerhalb des vertrauten Kulturkreises bekannt, das heißt sie sind im Sinne eines prozeduralen Wissensvorrats vorhanden, wobei betont werden muß, daß sie innerhalb des definitorischen Rahmens von procedural knowledge nicht unbedingt der Reflexion unmittelbar zugänglich sind, was wiederum heißt, daß es sich um Verfahren handelt, deren Analyse - zumindest für diejenigen, die sie anwenden - nicht ohne Schwierigkeiten ist. Die Reichweite solcher Schemata wird erst dann bewußt, wenn sie zu versagen scheinen oder ganz offensichtlich unangemessen sind, wobei dies nicht unbedingt eine interkulturelle Kommunikationssituation voraussetzt, sondern vermutlich innerhalb intrakultureller Kommunikation deutlich häufiger anzutreffen sein ist. Hier allerdings wird eben dieser Sachverhalt von den interagierenden Gruppen als natürlich - ja sogar notwendig angesehen, wobei intentionale Elemente - also das bewußte Inkaufnehmen der Unzulänglichkeiten - durchaus eine Rolle spielen dürften. Es existiert schlicht eine Anzahl 
von Individuen und Gruppen, mit denen man nicht oder nur dann kommuniziert, wenn es sich schlecht vermeiden läßt. Sollte es dennoch gezwungenermaßen zu einem Austausch kommen, stellen die Beteiligten oft mit Befriedigung fest, daß dieser schwierig und problematisch ist, sehen sich bestätigt und speichern diese Erfahrung unter der entsprechenden, bereits vorhandenen Rubrik, ab. Ein Abgleich der Systeme findet zwar statt, eine Kopplung und Parallelisierung jedoch nicht. Das Problem besteht nicht darin, daß diese Strategien nicht vorhanden sind, sondern darin, daß sie bewußt nicht angewandt werden: reduction of uncertainty wird nicht intendiert. Einer der Gründe scheint mir zu sein, daß, man sich zwar empathisch in die Rolle hineinversetzen kannn, daß man sie aber nicht sympathetisch interpretiert, da aus verschiedenen Gründen durch einen solchen Schritt eine Gefährdung der Identität erfolgen könnte.

Bei jungen Männern, die an geeigneten Stellen in den Fußgängerzonen deutschenr Städte in Gruppen herumstehen, muskulös und tätowiert, bereits um elf Uhr eine Dose Bier in der Hand und - bis vor kurzem jedenfalls - einen Kampfhund bei sich führend (nicht angeleint, versteht sich), verzichtet man, sofern man nicht zu ihnen gehört, gerne auf eine reduction of uncertainty. Es sind 'die Anderen' deren Rollen man über Stereotype hinreichend abgedeckt sieht. Empathie also durchaus, Sympathie jedoch keineswegs.

Kurz: innerhalb des eigenen, vertrauten Sprach- und Kulturraums ist Kommunikation a) nicht immer erfolgreich, da die oberflächlich wahrgenommene Bekanntheit eben nur eine scheinbare ist, b) bisweilen schon allein deshalb nicht möglich, weil kein Bedürfnis danach besteht, c) hin und wieder zum Scheitern verurteilt, weil a) und b) zutreffen. Die SympathieEmpathiefähigkeit sind, siehe das Zitat von Benson weiter oben, universal, ihre jeweiligen Objekte jedoch lokal.

\section{Die Exotismus-Strategie}

Bei der Kommunikation zwischen Mitgliedern unterschiedlicher Kultur- und Sprachkreise soll diese Unterscheidung nach Möglichkeit nivelliert werden. Neben den bereits erwähnten, rein technischen Aspekten wie Globalisierung, Migration und daraus resultierenden multikulturellen Gesellschaftsstrukturen, bei denen man in der Tat wünschen möchte, daß bestimmte Reibungsflächen nicht entstehen (vgl. Chen/Starosta 1998: 4ff), scheint auch eine bestimmte, durchaus als naiv zu bezeichnende Grundhaltung im Sinne eines allgemeinen Frieden stiftenden und völkerverbindenden Auftrages hinter diesem Anspruch zu stehen. Es geht also weitestgehend darum, den Anderen und sein Anderssein zu verstehen, wobei stillschweigend vorausgesetzt wird, daß dies vor allem im Falle des Zusammentreffens mit Angehörigen anderer Kulturen wichtig, notwendig und erstrebenswert ist. Neue, andere, möglicherweise sogar aufregende Erfahrungen werden in Aussicht gestellt. Nur: das Verstehen oder auch nur das Verstehenwollen ist häufig ein schwieriger und - weil oft nicht von Erfolg gekrönt - auch frustrierender Prozeß. Schon innerhalb des vertrauten Kulturkreises 
werden ja, wie bereits gesagt, Grenzen gezogen, jenseits derer keinerlei sympathetisches Verstehen mehr intendiert ist. ${ }^{6}$

Zweifelsohne hat jedoch das Zusammenführen von Repräsentanten verschiedener Nationen in einem interkulturellen universe of discourse eine sehr viel höhere Attraktivität als der Versuch, die Gäste des bis zum Überdruß berühmten Ballermann 6 und die Inhaber des Premierenabonnements der Staatsoper einander näher zu bringen. Soweit mir bekannt ist, hat es in diese Richtung auch keine Anstrengungen gegeben.

Eine der Antriebskräfte im Bereich des Interkulturaltätsparadigmas scheint der eingangs bereits erwähnte Exotismus zu sein, unter dem gemeinhin das Komplement zur Xenophobie verstanden wird. Das Konstrukt 'Fremdheit' wird aus der Exotismusperspektive nicht als identitätsbedrohend empfunden, sondern im Gegenteil, als erstrebenswertes Anderssein.

Bezüglich der Verteilung von Empathie und Sympathie läßt sich dann folgende schematische Verteilung annehmen: während bei Vorliegen von Xenophobie eine Empathie angenommen werden kann, die in einer möglicherweise völlig ungerechtfertigten Ablehung des Anderen mündet, ist für Exotismus Sympathie für das Andere eine Grundvoraussetzung. Diese Haltung kann unter Umständen dazu führen, daß die Andersartigkeit auch in Bereichen gesucht und gefunden wird, in denen prinzipiell durchaus Ähnlickeiten vorliegen (vgl. Culler 1988: 153ff). Sollte diese Annahme zutreffen, dann steht die interkulturelle Theorie und Praxis vor folgenden Problemen: sie muß, um ihren Gegenstand zu schaffen, das Andere akzentuiert als 'anders' herausarbeiten, sie muß in einem zweiten Schritt diese Andersartigkeit positiv markieren und sie muß dann die Strategien zur Verfügung stellen, die eine Annäherung, ein Verstehen, ermöglichen. Die grundsätzlichen Probleme, mit denen dieses Vorhaben konfrontiert ist, werden in der einschlägigen Literatur aus der Perspektive folgenden Positionen diskutiert: der logozentrische-universalistischen, der ethonzentrischrelativistischen und der egozentrisch-indivudualistischen. Der logozentrische Ansatz geht von der Annahme aus, daß menschliche Erfahrung - aufgrund der identischen Grundausstattung des homo sapiens - im Kern auch identisch ist. Die Welt wird von allen Individuen der Gattung letzlich in einer gewissen Gleichartigkeit wahrgenommen. ${ }^{7}$ Empathie wäre demnach nicht nur möglich, sondern unvermeidbar. Genau dies wird vom relativistischen Ansatz vehement bestritten. Erfahrung und damit auch Verstehen ist immer ein als soziales Konstrukt zu sehen. Die Welt, die wir erfahren, existiert nicht an sich, sondern sie wird konstruiert. Dementsprechend ist ein Verstehen ${ }^{8}$ von einer Position außerhalb dieses Konstrukts auch

\footnotetext{
${ }^{6}$ Das heroische Scheitern der Studenten, die im Gefolge der 68er Euphorie versuchten, die Arbeiterklasse bzw. das, was sie dafür hielten, zu verstehen, legt ein beredtes Zeugnis von den Problemen ab, die auch dann auftreten, wenn guter Wille vorhanden ist.

${ }^{7}$ Als Illustration mag das folgende Zitat von Pinker (1994:81f) dienen. "People do not think in English or Chinese or Apache; they think in a language of thought. [...] Knowing a language, then, is knowing how to translate mentalese into strings of words and vice versa."

${ }^{8}$ In aphorisitsch zugespitzter Form formuliert Bacon seine Gedanken zum Verstehen und zur zweifelhaften Rolle, die die Sprache seines Erachtens dabei spielt folgendermaßen: "Ganz deutlich zeigt sich die Herrschaft der alltäglichen, gar nicht auf wahres Erkennen und Lernen zielende Tendenz des Menschen, mit den Dingen und mit ihresgleich voreilig ins reine zu kommen an der ... Sprache. Die Antizipationen der Sprache halten den Geist am meisten besetzt und sind die lästigsten von allen. Nicht nur haben die Wörter von Haus aus einen Allgemeinheitscharakter, der oft über die Kontingenz und Inkohärenz ihrer faktischen Anwendung hinwegtäuscht, sondern die sprachliche Erfahrung der Dinge is primär unter der Direktive des auf ein flüchtiges ISSN 1615-3014
} 
nicht möglich. Humboldt, der hier exemplarisch zitiert werden soll formuliert diesen Standpunkt folgendermaßen:

"Durch denselben Akt, vermöge welches der Mensch die Sprache aus sich heraus spinnt, spinnt er sich in
dieselbe ein, und jede Sprache zieht um die Nation, welcher sie angehört, einen Kreis, aus dem es nur
insofern hinauszugehen möglich ist, als man zugleich in den Kreis einer anderen Sprache hinübertritt. Die
Erlernung einer fremden Spracheauf die richtige Art benutzt, ist daher die Gewinnung eines neuen
Standpunktes in der bisherigen Weltansicht, da jede das ganze Gewebe der Begriffe und der
Vorstellungsweise eines Teils der Menschheit enthält." (Humboldt (1936 V: 387f) Empathie ist unter diesen Voraussetzungen schwerlich vorstellbar. Dies widerspricht allerdings nicht nur jeder Alltagserfahrung, sondern würde auch in letzter Konsequenz auf eine statische Definition des Konstrukts 'Realität' hinauslaufen.

Der egozentrisch-individualistische Ansatz sucht die Aporien der beiden anderen zu vermeiden. Seine Annahme lautet, daß wir eben nicht alle gleich sind und von daher das Problem des Verstehens kein technisches ist, keines der Übesetzung des Pinkerschen mentalese in prinzipiell jede andere Sprache, daß wir auch nicht Gefangene der jeweiligen sozialen und kulturellen Konstrukte sind, sondern daß Individuen nur bis zu einem gewissen Grade soziale Bedeutungen kommunizieren und daß sie dementsprechend über eine Sphäre verfügen innerhalb derer sie ihre Identität autonom konstruieren und bestimmen (vgl. auch Harden/Witte 2002: 14ff). Diese Art der Aneignung der Welt geschieht dann,

when the speaker populates it [the word T. H] with his own intentions, his own accent, when he appropriates the word, adapting it to his own semantic and expressive intention. Prior to this moment of appropriation, the word does not exist in a neutral and impersonal language (it is not, after all, out of a dictionary that the speaker gets his words!), but rather it exists in other people's mouths, in other people's contexts, serving other people's intentions: it is from there that one must make the word, and make it one's own. (Bahktin 1986: 293f)

Mit anderen Worten, das Individuum ist zwar durch bestimmte Verfahren begrenzt, aber keineswegs so, daß es diese nicht in einer ganz spezifischen, idiosynkratischen Weise adaptieren oder assimilieren könnte. Der Ansatz entspräche weitestgehend dem weiter oben bereits angeführten Modell des Konstruktivismus. Man kann im Prinzip alles verstehen. Wenn man will! Das Wollen, das sympathetische Wahrnehmen des Anderen, ist jedoch der Dreh- und Angelpunkt, der in der Diskussion um interkulturelle Kommunikation kaum eine Rolle zu spielen scheint.

Vor diesem Hintergrund muß die von Edmondson/House gestellte Frage, ob das gesamte Konzept nicht möglicherweise überflüssig sei, gesehen werden. Ebenso die von Dittrich/Radke (1990) vorgebrachte Kritik hinsichtlich bestimmter Konzepte wie Ethnizität, ethnische und kulturelle Identität etc.. Nach Meinung der Autoren handelt es sich dabei um wissenschaftliche Konstrukte, die die wahren Konfliktursachen - wie z. B. fundamentale soziale Ungleichheit - verschleiern und auf eine Ebene heben, auf der mithilfe interkultureller Pädagogik im Prinzip unangemessene Lösungsmöglichkeiten geschaffen werden. Dies mag auf den ersten Blick als polemische Rhetorik erscheinen. Tatsache ist jedoch, daß das Betonen des jeweiligen Andersseins und die Reduzierung problematischer Kommunikation auf kulturelle Differenzen eine eingeschränkte und verzerrte Perspektive schafft. ${ }^{9}$ Die in diesem

Fertigwerden mit den Dingen bedachten praktischen Alltagsverstandes ... and deren wahren Beschaffenheit nur obenhin interessiert." (zit. nach Buck 1967:51)

${ }^{9}$ Vgl. auch Hamburger (1990), Schweitzer (1994) und Radtke (1995). Ein Überblick findet sich in Hu (2000: 75ff) eine positiv-kritsche Bestandsaufnahme bei Hinnenkamp (1994). 
Sinne bereits vorinterpretierte Wahrnehmung der Wirklichkeit wird auch von Hess $(1992: 1)^{10}$ als wenig fruchtbar und hilfreich emfunden:

Der Versuch, mit 'Kultur' als didaktischer Kategorie Unterricht zu planen oder zu analysieren, löst m. E. eben diesen Unterricht aus konkreten lebensweltlichen Bezügen und macht die Planung anfällig für bloße Stereotypisierungen. Genau diese Diskrepanz zwischen Fakt und Fiktion, von Realität und Interpretation die Etablierung von 'Kultur' als einer eigenständigen, separaten Bestimmungsgröße, die die Erfassung der Realität durch von außen vorgenommene Zuschreibungen und Metaphern ersetzt - war für mich das Hauptproblem.

Die von Hess kritisierte "Etablierung von 'Kultur' als einer eigenständigen Größe" schafft ja gerade die Vorausetzung für einen Typ von Exotismus, der dem Anderen unreflektiert positiv gegenübertritt, der das Verstehen jedoch - auf Grund der notwendigerweise stattfindenden Überhöhung - nicht unbedingt fördert.

Auf die weiter oben gemachten Ausführungen zu den Problemlösungsstrategien bezogen liegt dann der Schluß nahe, daß wenn das Problem im Bereich des Verstehens anderer Individuen verortet wird, seien sie nun Mitglieder des eigenen, wie auch immer definierten, sozialen Verbandes oder nicht, eben solche Strategien auch gefunden werden können. Reduction of uncertainty ist im Falle der Anderen zwar schwieriger, da das vorhandene prozedurale Wissen in einer solchen Situation nur eine begrenzte Reichweite hat, es ist aber keineswegs unmöglich; es ist allerdings auch nicht als bereits innate idea vorhanden, sondern muß erst geschaffen werden.

Adaption und Appropriation von Deutungschemata sind vor diesem Hintergrund also möglich und plausibel. Prozedurale Wissenssätze, Problemlösungstrategien könne erworben werden, jedoch nur dann, wenn eine problematische Situation bei ihrem Eintreten auch als solche erfahren und eine Lösung angestrebt wird.

Wittgensteins Reflexionen über Erfahrung und ihr Verhältnis zur Sprache sind in diesem Zusammenhang von ungeminderter Aktualität. ${ }^{11}$ In diesem Verständnis sind die Sätze der Grammatik $^{12}$ als Deutungsschemata, als Interpretationsverfahren anzusehen. In ihnen sind Erfahrungen gesammelt, die sich aus der Lebenspraxis ergeben. Bestimmte in dieser Form vorliegende Erfahrungen können als Dreh- und Angelpunkt unseres Denkens gesehen werden (vgl. §§ 146f). Sie sind als solche jedoch keineswegs dem Zwang zur Legitimation entzogen, sondern sie müssen sich in der Praxis bewähren. Wenn dies mißlingt, wenn die Praxis sich ändert und die Deutungsmöglichkeiten versagen, dann müssen neue Schemata geschaffen bzw. existierende modifiziert werden.

Man könnte sich vorstellen, daß gewisse Sätze von der Form der Erfahrungssätze erstarrt wären und als Leitung für die nicht erstarrten, flüssigen Erfahrungssätze funktionieren; und daß sich dies Verhältnis mit der Zeit änderte, indem flüssige Sätze erstarrten und feste flüssig würden.” ( $\$ 96)$

\footnotetext{
${ }^{10}$ Ganz in diesem Sinne argumentiert auch Donald (1988:32) mit dem Hinweis auf „the apparatuses of discourse, technologies and institutions (print, capitalism, education, mass media and so forth) which produced what is generally recognised as 'the national culture' ... the nation is an effect of these cultural technologies, not their origin. A nation does not express itself through its culture: it is the cultural apparatuses that produce 'the nation' What is produced is not an identity or single consciousness ... but (hierarchically organised) values, dispositions and differences. This cultural and social heterogeneity is given a certain fixity by the articulating principle of 'the nation'. The 'national' defines the culture's unity by differentiating it from other cultures, by marking the bounderies; a fictional unity, of course, because the 'us' on the inside is itself always differentiated."

${ }^{11}$ Die folgenden Angaben beziehen sich auf Wittgenstein, L. (1970), Über Gewißheit.

${ }^{12} \mathrm{Zu}$ Wittgensteins Grammatikverständnis vgl. seine Ausführungen in Über Gewißheit §§ 341-343.
} 
Dieser Situation sieht sich mit Sicherheit der Benutzer einer Fremdsprache ausgesetzt. Problem solving wäre für ihn das Verflüssigen der Erfahrungsätze bzw. das Erstarrenlassen bisher flüssiger Sätze. Das Individuum verfügt im Normalfall über hinreichende Problemlösungsverfahren, um die meisten kommunikativen Situationen adequat zu bewältigen. Die Reduktion der Unsicherheit, die sympathetische Annäherung an den Anderen, die unter eben diese Strategien fällt, wird allerdings nur dann vorgenommen, wenn dadurch in der Tat eine als problematisch empfundenen Situation gelöst werden kann. Eben weil die Individuen über empathische Fähigkeiten verfügen, weil sie sich in die Situation, Position oder Rolle des Anderen hineinversetzen können, werden sie nicht diesem nicht unbedingt mit Sympathie entgegenkommen.

\section{Conclusio}

Wenn man von der Richtigkeit der bisher gemachten Ausführungen ausgeht, dann kann Kommunikationstraining im Allgemeinen und interkulturelles Kommunikationstraining im Besonderen nur auf Empathiefertigkeit und -fähigkeit hinauslaufen. Es birgt durchaus die Gefahr, einfach nur manipulatives Verhalten zu erzeugen, denn Sympathie entzieht sich einem curricular gesteuerten Lernprozeß. Die Annahme, daß Verstehen mit Sympathie gekoppelt ist, läßt sich kaum aufrechterhalten. Fertigkeiten, die in diesem Bereich erworben werden, führen nicht notwendigerweise zu einer sympathetischen Interpretation der Rolle des Anderen. Das potentielle Moment der Bedrohung der Identität oder auch nur materieller Errungenschaften wird durch eine Erweiterung des Verhaltensrepertoirs nicht weniger bedrohlich.

Ein grundsätzliches Problem der interkulturellen Kommunikation besteht demnach darin daß Annahmen bezüglich der an einem solchen Prozeß Beteiligten gemacht werden, die nicht notwendigerweise zutreffen. Die Identifikation des 'Anderen' als weitgehend kulturspezifisch determiniertes Anderssein läßt das Ähnliche in den Hintergrund treten. Die Konzentration auf Unterschiede und die Annahme daß deren wechselseitiges Verstehen eine sympathetische Akzeptanz erzeugt, führt tendenziell dazu, Unterschiede als prinzipiell positiv darzustellen und so die lebensweltlichen Probleme, die dadurch hervorgerufen werden, als sekundär erscheinen zu lassen. Ablehnung, die das Individuum aus welchen Gründen auch immer, empfinden mag, ist so gesehen immer nur auf mangelndes Verstehen zurückzuführen. Kehren wir kurz zur Bibel zurück. Das Gebot der Nächstenliebe ist, verglichen mit den mosaischen Geboten, relativ jung. Es steht aber in ihrer Tradition. Gebote sind Regulative in Bereichen, die dieser offensichtlich Bedürfen. Man kann also davon ausgehen, daß den Nächsten zu lieben nicht etwas ist, das der menschlichen Natur sui generis zu eigen ist, ebensowenig wie das Unterlassen des Tötens, des Ehebrechens und des Begehrens des Nächsten Hab und Gut. Gebote rekurrieren in ihrer klaren Schlichtheit auch nicht auf ein rationales Durchdringen der Materie und sie vermeiden bewußt den Appell an das Gute. Insofern sind sie weit realistischer und alltagstauglicher als jedweder Versuch, durch Verstehen letztendlich Sympathie zu erzeugen. Von Lot wissen wir ja auch nur, daß er ein Gebot befolgte, daß er seinen Gästen gegenüber sympathetisches Verstehen empfunden hat, wird nicht gesagt. Es kann, angesichts deren wirklich exotischer, da himmlischer Natur, mit Fug und Recht bezweifelt werden. 


\section{Literaturangaben}

Anderson, J. R. (1976): Language, Memory, and Thought. Hillsdale / N. J.

Bakhtin, M. M. (1986): Speech Genres and Other Late Essays. (Transl. by V. W. McGee). Austin.

Benson, C. (2001): The Cultural Psychology of Self. London.

Berger, C. R. / Bradac, J. J. (1982): Language and Social Knowledge. Uncertainty in Interpersonal Relations. London.

Buck, G. (1967): Lernen und Erfahrung. Stuttgart.

Chen, G.-M. / Starosta, W. J. (1998): Foundations of Intercultural Communication. Boston.

Coupland, N. / Giles, H. / Wiemann, J. M. (eds) (1991): "Miscommunication" and Problematic Talk. London.

Culler, J. (1988): Framing the Sign. Criticism and its Institutions. Oxford.

Dittrich, E. J. / Radtke, F.-O. (eds) (1990): Ethnizität. Wissenschaft und Minderheiten. Opladen.

Donald, J. (1988): "How English is it? Popular literature and national culture". New Formations 6: 31-47.

Edmondson, W./House, J. (1998): "Interkulturelles Lernen: ein überflüssiger Begriff". Zeitschrift für Fremdsprachenforschung 9/2:161-188.

Enzensberger, H. M. (1968): "Eine Theorie des Tourismus". In: Ders. (1968): Einzelheiten. Frankfurt/M: 149-167.

Esser, Hartmut (1996): "Ethnische Konflikte als Auseinandersetzung um den Wert von kulturellem Kapital". In: Heitmeyer, W. / Dollase, R. (eds): Die bedrängte Toleranz. Ethnischkulturelle Konflikte, religiöse Differenzen und die Gefahren politisierter Gewalt. Frankfurt/M.: 64-99.

Gordon, G. (1969): The language of communication: A logical and psychological approach. New York.

Hamburger, F. (1990): Der Kulturkonflikt und seine pädagogische Kompensation. In: Dittrich, E. J. / Radtke, F.-O. (eds) (1990): Ethnizität. Wissenschaft und Minderheiten. Opladen.: 311-328.

Harden, Th. (1983): Die subjektive Modalität in der zweiten Sprache. Frankfurt.

Harden, Th. (2000): "The Limits of Understanding". In: Harden, Th. / Witte, A. (eds) (2000): The Notion of Intercultural Understanding in the Context of German as a Foreign Language. Oxford:103-125.

Harden, Th. / Witte, A (eds) (2000): The Notion of Intercultural Understanding in the Context of German as a Foreign Language. Oxford.

Harden, Th. / Witte, A. (2000): "Introduction". In: Harden, Th. / Witte, A. (eds) (2000): The Notion of Intercultural Understanding in the Context of German as a Foreign Language. Oxford: 7-27.

Heider, F. (1958): The Psychology of Interpersonal Relations. New York.

Heijl, P. M. (1987): "Konstruktion der sozialen Konstruktion: Grundlinien einer konstruktivistischen Sozialtheorie". In: Schmidt, S. J. (ed) (1987): Der Dikurs des radikalen Konstruktivismus. Frankfurt/M.: 303-340.

Hess, H.-W. (1992): Die Kunst des Drachentötens. Zur Situation von Deutsch als Fremsprache in der Volksrepublik China. München.

Hinnenkamp, V. (1994): "Von den Schwierigkeiten (mit) der Interkulturellen Kommunikation". Sprache und Literatur 74: 3-17.

$\mathrm{Hu}$, A. (2000): "Intercultural Learning and its Difficult Aspects - An Analysis of the Criticism in Relation to a Controversial Concept". In: Harden, Th. / Witte, A (eds) (2000): The Notion of Intercultural Understanding in the Context of German as a Foreign Language. Oxford: 75-103. 
Humboldt, W. v. (1936): "Grundzüge des allgemeinen Sprachtypus". In: Wilhelm von Humboldt: Gesammelte Werke Bd. V, ed. von Albert Leitzmann, Bruno Gebhardt, Wilhelm Richter. Berlin.

Leech, G. (1983): The Priciples of Pragmatics. London.

Maturana, H. R. (1982): Erkennen: Die Organisation und Verkörperung von Wirklichkeit. Braunschweig.

Morley, D. / Robins, K. (1995): Spaces of Identity. London.

Parisi D. / Castelfranchi, C. (1981): "A goal analysis of some pragmatic aspects language". In: Parret, H. / Sbisá, M. / Verschueren, J. (eds) (1981): Possibilities and Limitations of Pragmatics. Amsterdam: 551-568.

Pinker, S. (1994): The Language Instinct. The New Science of Language and Mind. London.

Radtke, F.-O. (1995): "Interkulturelle Erziehung. Über die Gefahren eines pädagogisch halbierten Anti-Rassimus". Zeitschrift für Pädagogik 41: 853-864.

Roth, G. (1987): "Erkenntnis und Realität: Das reale Gehirn und seine Wirklichkeit". In: Schmidt, S. J. (ed) (1987): Der Diskurs des radikalen Konstruktivismus. Frankfurt/M.

Samovar, L. A. / Porter, R. E. (eds) (1988): Intercultural Communication: A Reader. Belmont / CA.

Schlesinger, P. (1987): "On national identity: some conceptions and misconceptions criticised". Social Science Information 26/2: 219-264.

Schweitzer, H. (1994): Der Mythos vom interkulturellen Lernen. Hamburg.

Smith, A. (ed) (1966): Communication and Culture: Readings in the Codes of Human Interaction. New York.

Todorov, T. (1984): The Conquest of America: the Question of the Other. New York. Winston, P. H. (1977): Artificial Intelligence. Reading/Mass.

Witte, A. (2000): "How to be an Alien - Learning a Foreign Language and Understanding Culture". In: Harden, Th. / Witte, A (eds) (2000): The Notion of Intercultural

Understanding in the Context of German as a Foreign Language. Oxford: 53 - 75.

Wittgenstein, L. (1970), Über Gewißheit. Frankfurt. 